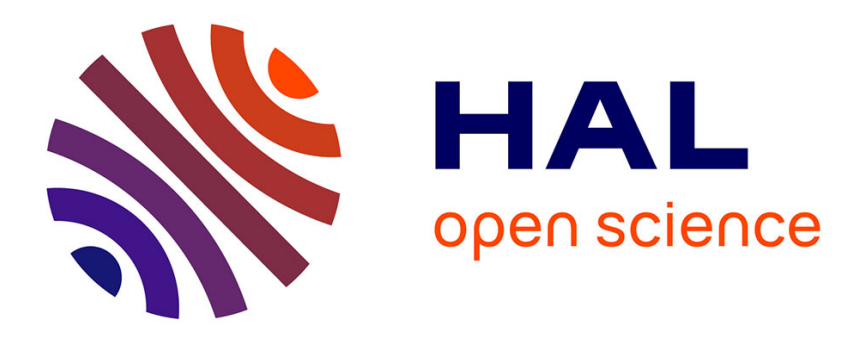

\title{
Wnt/Planar Cell Polarity Signaling: New Opportunities for Cancer Treatment
}

\author{
A. Daulat, J.P. Borg
}

\section{To cite this version:}

A. Daulat, J.P. Borg. Wnt/Planar Cell Polarity Signaling: New Opportunities for Cancer Treatment. Trends in Cancer, 2017, 3 (2), pp.113-125. 10.1016/j.trecan.2017.01.001 . hal-01790716

\section{HAL Id: hal-01790716 https://hal.science/hal-01790716}

Submitted on 13 May 2018

HAL is a multi-disciplinary open access archive for the deposit and dissemination of scientific research documents, whether they are published or not. The documents may come from teaching and research institutions in France or abroad, or from public or private research centers.
L'archive ouverte pluridisciplinaire HAL, est destinée au dépôt et à la diffusion de documents scientifiques de niveau recherche, publiés ou non, émanant des établissements d'enseignement et de recherche français ou étrangers, des laboratoires publics ou privés. 
3

4

5

6

7

8

9

10

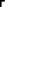

17 19 0
Wnt/Planar Cell Polarity signaling:

new opportunities for cancer treatment

Avais M. Daulat ${ }^{1}$ and Jean-Paul Borg ${ }^{1}$

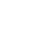

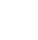

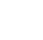

$1{ }^{1}$ Centre de Recherche en Cancérologie de Marseille, Aix Marseille Univ UM105, Inst Paoli Calmettes, UMR7258 CNRS, U1068 INSERM, «Cell Polarity, Cell signalling and Cancer Equipe labellisée Ligue Contre le Cancer », Marseille, France

* To whom correspondence should be addressed: jean-paul.borg@inserm.fr/Phone 33-48697-7201, Fax 33-4-8697-7499

Keywords: Wnt pathway, non-canonical, planar cell polarity, signaling, therapeutic targets 


\section{Abstract}

23 Cancer cells are addicted to a large spectrum of extracellular cues implicated in the initiation, 24 stem cell renewal, tumor growth, dissemination in the body, and resistance to treatment. 25 Among these factors, Wnt ligands and their associated signaling cascades contribute to most of these processes, paving the way for opportunities in therapeutic development. The developmental Wnt/Planar Cell Polarity pathway is the most recently described branch of Wnt signaling for having a strong implication in cancer development at early and late stages. We will depict here some of the latest knowledge accumulated on this pathway and the pending questions, present the most convincing findings about its role in cancer and review the most promising strategies currently designed to target its components. 


\section{Not one but several Wnt signaling pathways}

Among the developmental signaling pathways defective in cancer (Hedgehog, Notch, Hippo, Wnt), it recently became clear that Wnt signaling plays a pivotal role in all steps of the disease, from tumor initiation to cancer growth and dissemination. During the past three decades since the discovery of the first Wnt ligand and its implication in cancer (BOX 1) [1, 2], a large number of genetic, functional and translational studies have greatly improved our knowledge on this pathway. In particular, many important Wnt signaling components have been identified and functionally characterized including a network of 19 Wnt ligands, 10 membrane Frizzled receptors (Frizzled) or co-receptors $(\mathrm{LRP} 5 / 6, \ldots)$ that trigger intracellular cascades with multiple outputs [3-5] (Figure 1A). Part of the challenge lies in the complexity of the pathway in which multiple combinatory interactions are generated by the intervening components at the cell surface and inside the cells. Painting a simplistic picture of the pathway, Wnt signaling is classically divided in two branches whose delineation relies on differential requirement for $\beta$-catenin, an armadillo-repeat cytoplasmic adaptor protein with membrane and nuclear functions. For many years, Wnt/ $\beta$-catenin signaling (also referred as canonical Wnt signaling) has been the most extensively characterized pathway at the molecular level and in human diseases. This has led to major discoveries about its mode of action and its prominent implication in cancer (for review, see [6]). The $\beta$-cateninindependent Wnt pathways (hereafter named non-canonical pathways) utilize a different panel of Wnts and (co)receptors compared to the canonical pathway although some Wnt components such as Dishevelled are shared by both pathways. However, depending on the cellular context and expression of particular co-receptors, a given Wnt can activate either $\beta$ catenin-dependent or $\beta$-catenin-independent Wnt signaling. The non-canonical Wnt pathway is often seen as subdivided in two branches, one using intracellular calcium as a second messenger $\left(\mathrm{Wnt} / \mathrm{Ca}^{2+}\right)$ and the other one defined by a conserved genetic pathway (Wnt/Planar 
Cell Polarity or PCP) (Figure 1). However, as discussed below, one can envision these two branches as a single one on the basis of biochemical arguments. Recent reviews have depicted canonical and Wnt/Ca2+ pathways in great details (see for example [3]). In this review, we will focus on the Wnt/PCP pathway whose contribution in many cancers has been recently uncovered.

\section{The evolutionary conserved Planar Cell Polarity}

In 1982, Gubb and Garcia-Bellido studied mutants of the fruit fly (Drosophila melanogaster) harboring defects in the orientation of hairs and sensory bristles on the wings, describing for the first time what is now routinely called PCP defects [7]. PCP refers to the organization of the epithelium orthogonal to the apico-basal polarity axis which orients epithelial cells attached to extracellular matrices. Genetic studies in flies further identified a group of genes, so-called core PCP genes, responsible for the establishment and maintenance of PCP in wings and ommatidia $[8,9]$. The encoded molecules are evolutionary conserved and are members of diverse family of receptors (Flamingo, Frizzled, Vang-Gogh-like, Ptk7, Ryk, Ror2, Daschous, Fat) and cytoplasmic adaptors (Dishevelled, Prickle, Diego). Asymmetric distribution of PCP components in polarized cells, - Vangl-like, Prickle and Diego localize at the anterior sides of the plasma membrane and Frizzled, Dishevelled and Dachsous at the posterior sides of the plasma membrane -, is inherent to PCP functions, and is achieved through diverse and not completely understood molecular mechanisms [10]. PCP components are highly conserved throughout evolution, from drosophila to humans, albeit one fruit fly gene has usually several paralogs in vertebrates. For example, the drosophila Vang-Gogh and Prickle1 genes have two (Vangl1 and Vangl2) and four (Prickle1-4) mammalian paralogs, respectively. In the mouse, PCP is required for orientation of stereocilia in the cochlea, orientation of hairs in the skin and morphogenesis of many organs [11-13]. In the early 2000s, PCP molecules were found 
implicated in the regulation of convergent extension (C-E), a process characterized by cellular movements controlled by JNK and RHO activities required at early stages of development (gastrulation), and in ciliogenesis in vertebrates $[3,8,10,13-15]$. The central role of PCP in vertebrate development is further evidenced by the dramatic impact of misexpression of its components in embryos, leading to neural tube defects, - the most obvious and dramatic being craniorachischisis in mice and humans mutant for Vangl-like -, and to abnormal formation of the body axis during gastrulation [16]. Work from many laboratories has assigned PCP to a non-canonical Wnt pathway referred thereafter as Wnt/PCP pathway.

\section{Wnt/PCP is a $\beta$-catenin independent Wnt pathway}

Wnts (Wingless/Int-1) are secreted glycoproteins expressed during embryonic development and throughout adult life, acting in diverse processes such as cell proliferation, stem cell maintenance, cell migration, survival, and cell fate determination. In vertebrates, Wnts can activate $\beta$-catenin-dependent and -independent pathways, however genetic studies conducted in Drosophila concluded that Wingless, the sole fly Wnt, does not control PCP [8]. Organization of Wnt pathway is very complex owing to the important number of Wnt ligands and membrane receptors of the seven transmembrane Frizzled family or single-spanning transmembrane (co)receptors able to enter into multiple $\mathrm{Wnt} /(\mathrm{co})$ receptor combinations at the plasma membrane $[3,17]$. As mentioned above, use of $\beta$-catenin defines the canonical and best studied Wnt pathway (BOX 2) [3]. Depending on the cellular context, Wnt/PCP pathway can be triggered by various Wnts including some which generally activate the $\beta$-catenin dependent pathway. There is a large body of evidence that shows how the presence of coreceptors at proximity of Frizzled receptors orients the decision toward either $\beta$-catenin dependent or independent pathways. However, among the Wnts, WNT-5A, WNT-7 or WNT11 are known to mostly favor Wnt/PCP pathway. In parallel, it is commonly admitted that 
FRIZZLED-3, -6 and -7 mostly orient toward Wnt/PCP signaling whereas canonical Wnt

110 pathway usually utilizes FRIZZLED-1 and -4 . Wnt co-receptors such as tyrosine kinase receptor family members (PTK7, ROR2, RYK) or membrane proteins (CD146, VANGL2, Syndecan, Glypican) have a demonstrated implication in Wnt/PCP pathway [18, 19]. They can directly bind to Wnts (CD146, ROR2, RYK) or facilitate interaction between Wnts and their cognate Frizzled receptors. For example, formation of a Wnt-Frizzled-ROR2 complex triggers Wnt/PCP signaling during development and in cancer cells. The ability of ROR2 to heterodimerize with PTK7, another tyrosine kinase receptor, and to transduce downstream events on its own adds additional layers of complexity to this signaling platform [20-22]. Furthermore, oligomerization of Frizzled receptors is not only necessary for their maturation and membrane localization [23] but also represents, as demonstrated for other seven transmembrane proteins $[24,25]$, an opportunity for the recruitment of signaling molecules implicated in canonical or non-canonical Wnt pathways, increasing again system complexity. Binding of Wnt ligands leads to recruitment of Dishevelled to Wnt/PCP Frizzled receptors and activation of small GTPases of the Rho family (Cdc42, Rac1, RhoA) involved in actin cytoskeleton remodeling and cell contractility, and JNK pathway [14]. In vertebrates, $\beta$ catenin independent signaling is traditionally divided into $\mathrm{Wnt} / \mathrm{Ca}^{2+}$ and $\mathrm{Wnt} / \mathrm{PCP} \mathrm{JNK}$ dependent signaling which can be both activated by Wnt5a. However, in Xenopus, Randall Moon's team showed that Prickle1 and Dishevelled can trigger JNK (monitored by AP1 reporter assays) and calcium (increase of $\mathrm{Ca}^{2+}$ dynamics and activation of CAMKII and PKC, two $\mathrm{Ca}^{2+}$ dependent kinases) signaling, leaving the possibility open that $\mathrm{Wnt} / \mathrm{Ca}^{2+}$ and Wnt/PCP branches function as one signaling pathway or, at least, that they cross-talk [26, 27]. None of these biochemical assays have been applied to Drosophila where PCP signaling was genetically, not biochemically, defined. Together, these data suggest subtle regulation and overlap between these two Wnt pathways. 
Canonical Wnt and non-canonical Wnt/PCP pathways are engaged in intimate relationships by sharing common components such as Dishevelled, PTK7 and RYK which can positively or negatively act on both signaling cascades $[14,15]$. For example, Wnt-5a can trigger $\beta$-catenin dependent or independent pathway $[28,29]$ and PTK7 can directly interact with $\beta$-catenin, promoting a canonical Wnt signaling during Xenopus development, or activate Wnt/PCP signaling [20, 30-32]. Finally, like most Wnt/PCP components, Prickle behaves as an inhibitor of $\beta$-catenin dependent signaling $[3,33]$.

\section{Deregulation of Wnt/PCP signaling in cancer}

Whereas loss of Wnt/PCP signaling, mostly by gene mutations, is linked to human genetic disorders (defects of neural tube closure or palate cleft, kidney diseases, ciliopathies) (for reviews see $[8,9,14])$, up-regulation of Wnt/PCP components is observed in many cancers, this event being most of the time, although not exclusively, associated to a poor prognosis (Table 1). Recent studies have highlighted how these deregulations are associated to the classical features of cancer progression. We will describe thereafter how Wnt/PCP proteins are involved in processes such as cell proliferation, stemness, epithelial-mesenchymal transition (EMT), cell migration, tumor invasiveness and resistance to treatment (Figure 1B).

\section{Cancer cell proliferation and stemness}

Involvement of canonical Wnt signaling in the early stages of colon cancer is well established as mutations in up to $90 \%$ of colorectal cancers (CRCs) of APC (80\%), $\beta$-catenin $(10 \%)$ or AXIN which constitutively activate the pathway were found [3]. A recent study analyzing a large cohort of non-metastatic CRC patients showed that enhanced expression of noncanonical Wnt-5a confers a better clinical outcome [34]. Accordingly, xenografts of colorectal HCT116 cells overexpressing Wnt-5a are less proliferative and less tumorigenic [34]. This tumor suppressing role of Wnt-5a has also been described in other cancer types (lymphoma, 
thyroid cancer) [35-37] and is due to inhibition of canonical Wnt pathway [29]. Paradoxically, in other studies, Wnt-5a is described as a poor prognosis marker for patients suffering from CRC and gastric cancer and behaves as a pro-migratory and pro-invasive ligand [38, 39]. Along the same line, downregulation of the Wnt/PCP receptors PTK7 and VANGL1/VANGL2 impairs cell proliferation of colon and breast cancer cells, respectively [40-43]. A recent report showed that FRIZZLED-7 along with other Wnt/PCP components is upregulated in ovarian cancer cells conferring cell proliferation, cell cycle progression, and stemness mediated in part by RhoA activity [44]. The PDZ protein SCRIBBLE plays a prominent role in apico-basal polarity and behaves as a tumor suppressor in Drosophila [45]. Its function has somehow diverged along evolution as it participates to Wnt/PCP signaling in vertebrates [46]. However, its tumor suppressing function is conserved in breast and prostate cancer through an increase of cancer cell survival [47, 48], MAPK and Hippo pathways which promote polarity defects, cancer cell proliferation and invasiveness, self-renewal and tumorigenic capacities of cancer stem cells [48-51]. In stark contrast with canonical Wnt pathway, little is known about the transcriptional events and target genes of Wnt/PCP signaling which makes its study difficult. However, GRHL3, a transcription factor involved in epidermal wound repair and acting as a tumor suppressor in squamous cell carcinoma, represents a notable exception [52, 53]. GHRL3 is the orthologous to Drosophila Grainy head (Grh), a protein involved in PCP signaling in wing hair formation and ommatidium orientation in flies [54, 55]. Mechanistically, human GRHL3 controls the expression of RhoGEF19, an activator of the small GTPase family RhoA, leading to actin polymerization, cytoskeletal rearrangement and directed migration in wound healing [52]. Recently, Jarid2, a developmental transcription factor member of the Polycomp Repressor complex 2, has been shown to control expression of Wnt/PCP components such as Wnt-9a, Frizzled-2, and Prickle1 which are important for stemness of murine embryonic stem cells [56]. Such a causal 
link has not been yet established in cancer stem cells; however human JARID2 promotes EMT in hepatocellular, lung and colon cancer cells [57, 58]. Lastly, PTK7 was recently described as a hematopoietic and colon cancer stem cell marker whose function has yet to be determined $[59,60]$.

\section{Epithelial-Mesenchymal Transition}

EMT is a physiological reversible process which describes the transition of epithelial cells from a polarized to a mesenchymal state prone to motility. It occurs during normal embryonic development but also in tumors leading to increased invasion and drug resistance, and is controlled by transcriptional programs driven by many signaling pathways [61]. Upregulation of Wnt-5a has been observed in cellular systems undergoing EMT such as squamous carcinoma cells [62] and Madin-Darby Canine Kidney (MDCK) cells treated with TGF $\beta$ [63]. Levels of FRIZZLED-4 and PTK7 receptors are also high in prostate cancer, and in MDCK or human embryonic stem cells in which EMT is triggered [63-65]. Cause or consequence? In melanoma cells, Wnt-5a promotes EMT through a PKC-dependent mechanism, related to $\mathrm{Wnt} / \mathrm{Ca}^{2+}$ and possibly to $\mathrm{Wnt} / \mathrm{PCP}$, which leads to expression of mesenchymal markers such as Snail and Vimentin and decreases E-cadherin expression [66]. Recent results attribute to SCRIBBLE a direct role in EMT in normal [67] and tumoral [68] situations through the modulation of SMAD3/SMAD4 and MAPK-ERK activities, respectively. On the other hand, induction of EMT in breast cancer cells delocalizes SCRIBBLE from the plasma membrane inducing a Hippo-dependent pro-metastatic program [50]. Recently, elevated Wnt-5a/Wnt-5b and FRIZZLED-2 levels have been observed in several types of metastatic cancers (liver, lung, colon, and breast) expressing EMT markers. In this study, FRIZZLED-2 is a cornerstone and targetable molecule driving EMT and metastasis through FYN and STAT3, two novel components of Wnt pathways [69].

\section{Cell migration and invasiveness}


Extensive data have been accumulated on the role of $\mathrm{Wnt} / \mathrm{PCP}$ deregulation in tumoral

210 dissemination through in vitro and in vivo studies [40, 41, 43, 49, 66, 70-73]. In skin cancers such as melanoma, overexpression of Wnt-5a correlates with enhanced cell invasion and metastasis, and with poor outcome [73]. These effects can be mediated by different Wnt receptors including FRIZZLED-3, -5 or ROR2 [73-76]. At the cellular level, stimulation of melanoma cells by Wnt-5a leads to an asymmetrical accumulation of FRIZZLED-3 in intracellular Wnt-mediated receptor-actin-myosin polarity (W-RAMP) structures under the control of two small GTPases, Rab4 and RhoB [77]. Mechanistically, W-RAMPs localize at the trailing edge of migrating cells where they locally activate PKC leading to increased contractility and focal adhesion disassembly rates [78]. Wnt-5a can also control focal adhesion dynamics through FRIZZLED-2, APC and Dishevelled [79]. APC and Dishevelled associate with Focal Adhesion Kinase (FAK) and PAXILLIN which are important for Wnt5a-dependent focal adhesion turnover. This particular pathway seems initiated through an association between Wnt-5a/FRIZZLED-2 and integrins at the leading edge of migrating cells. In another study, single molecule RNA sequencing of circulating tumor cells (CTCs) from prostate cancer revealed an increase of Wnt-2 driven non-canonical signaling which suppresses anoikis and increases cell motility [80].

Genomic analysis led to the stratification of breast cancers into five main classes according to gene expression. Triple negative breast cancers (TNBCs) are the most aggressive entity, lacking expression of HER2 and estrogen/progesterone receptors, with no yet available targeted therapy. Recent data from our lab found that PRICKLE1 and VANGL2 are both overexpressed in TNBCs [43, 70] whereas high expression of VANGL1, a VANGL2 homologue, was associated to poor prognosis in estrogen receptor-positive breast cancer patients [81]. PRICKLE1 which is known to interact with VANGL2 [82] is implicated in focal adhesion dynamics [70, 71, 83]. Interestingly, PRICKLE1 downregulation has been 
reported to strongly impair cell motility via three possible downstream regulators, Akt [70], and Rho-GEF [83] in TNBC cells or LL5 $\beta$ [71] in other cell systems. Moreover, activation of surviving Akt-dependent mechanism by $\mathrm{Wnt} / \mathrm{PCP}$ proteins contributes to cancer progression $[70,84]$. Since the first observations that elevated amounts of Wnt-5a are found in invasive breast cancers [85] [86], other Wnt/PCP-related molecules have also been proposed as markers of aggressiveness. This is for instance the case of two other Wnt ligands, Wnt-7a and Wnt-11 [72, 87], as well as receptors (FRIZZLED-2, -7, VANGL2) [43, 69] and adaptors (SCRIBBLE, PRICKLE1) [42, 70, 88]. In TNBCs, VANGL2 is thought to act downstream of ROR2 [89] or Frizzled [90] and its overexpression appears to correlate with JNK pathway activation through a direct interaction with the p62/SQSTM1 scaffold [43]. VANGL2 may also promote cell migration through interaction with SCRIBBLE [41]. Altogether these data suggest that VANGL2 act as signaling platform able to mediate downstream signaling events through its interaction with different scaffold proteins (SCRIBBLE, p62/SQSTM1 or PRICKLE1). The finding that, in neuroblastoma and fibrosarcoma cells, PRICKLE1 and VANGL2 act as tumor suppressor genes [91, 92] indicates that their implication in cancer might vary depending on the cellular context.

First identified in colon cancer cells $[93,94]$, PTK7 has since been shown to behave as a poor prognosis marker in colon cancer patients and to contribute to the metastatic program [40, 95]. This inactive tyrosine kinase receptor is a Wnt-5a co-receptor which forms heterodimers with ROR2 and mediates JNK activation [20, 21]. In other contexts, PTK7 can modulate $\beta$ catenin dependent Wnt pathway [30, 31], induce cell migration $[40,96]$ and associate with diverse cancer-related membrane receptors (VEGFR1, Plexins) [95]. Moreover, upregulation of PTK7 has been reported in Acute Myeloid Leukemia (AML) and $\mathrm{T}$ cell acute lymphoblastic leukemia (T-ALL) [96, 97]. High PTK7 levels in AML cells are associated with low circulating blast cell counts most probably because of retention of PTK7 positive 
cells in the bone marrow [59]. This may well explain the resistance of AML-PTK7 positive patients to chemotherapy as this treatment aims at eliminating circulating cycling blast cells [96]. Other Wnt/PCP components (Wnt-5a, PRICKLE1, VANGL2, CELSR1, FRIZZLED-3,

-7) have been also reported to be poor prognosis markers in chronic lymphocytic leukemia (CLL) when overexpressed [98, 99]. Autocrine release of Wnt-5a by CLL cells stimulates ROR1, a ROR2 homologue, and controls the chemotactic response and proliferation by inducing ROR1/ROR2 heterodimerization which activates RhoA and Rac1 [98-100]. This proliferative pathway can be targeted with specific antibodies inducing apoptosis [101].

\section{Wnt/PCP pathway in host-tumor interaction}

Tumor growth and dissemination is not only governed by genetic alterations of cancer cells but also by an altered deregulated communication with adjacent tissues. In Hodgkin lymphoma, canonical Wnts were shown to be secreted by the endothelial vasculature, promoting chemotaxis and adhesion of lymphoma cells [102]. In prostate cancer, genotoxic treatment leads to WNT16B secretion by the tumor microenvironment which initiates a paracrine activation of canonical Wnt signaling in cancer cells and drug resistance [103]. Wnts are secreted, lipid-modified glycoproteins whose maturation in the endoplasmic reticulum relies on Porcupine, an eight transmembrane spanning acyl transferase [28]. Inhibition of Porcupine was thus proposed as a strategy to repress Wnt production and treat squamous cell carcinoma and CRCs (Table 2). Several routes involving protein carriers, filipodia or cytomenes and extracellular vesicles such as exosomes are involved in the extracellular transport of Wnts in the hydrophilic extracellular space to generate signaling gradients in tissues [104]. Exosome are 30-150nm vesicles that allow the transfer of proteins, lipids and genetic materials and play a key role in intercellular communication between cancer cells and their microenvironment [105]. In melanoma, Wnt-5a has been shown to 
induce a calcium dependent release of exosomes that contain IL-6, VEGF and MMP2. This process stimulates the adjacent endothelial cells and favor invasiveness of cancer cells to distant tissues [106]. Recent reports have highlighted the role of Wnt/PCP pathway in cancerassociated fibroblasts (CAFs) which promote breast cancer metastasis. Indeed, Luga et al. have shown that CAFs generate exosomes which are internalized by cancer cells, loaded with Wnt-11, and released by an autocrine loop promoting metastasis through VANGL1, Dishevelled and PRICKLE1 [72]. Conversely, breast cancer cells can secrete Wnt-7a which activates CAFs and promotes invasion through a TGF $\beta$-dependent signaling [87]. Wnt/PCP pathway thus plays an important role in tumor-CAFs communication for the regulation of cell motility, invasion and niche formation.

\section{Wnt/PCP pathway in resistance to treatment}

Wnt/PCP can intervene by different mechanisms on drug resistance either indirectly (for example through its participation to EMT) or directly. In melanoma, hypoxia induces Wnt-5a expression and contributes to resistance to B-RAF inhibitors [84, 107]. The latter mechanism occurs through ROR2 or FRIZZLED-7, RYK and the pro-survival Akt pathway which blocks apoptosis. Elevation of PRICKLE1 expression in poor prognosis breast cancer is correlated with increased Akt activation; however its contribution to drug resistance has not been evaluated [70]. Overexpression of PTK7 is associated with drug resistance through retention of blasts in the bone marrow of AML patients and to resistance anthracycline-based chemotherapy by an unknown mechanism in breast cancer patients [96, 108]. Recently, Wnt/PCP signaling has been shown to be elevated in prostate circulating tumour cells resistant to treatment with enzalutamide, an androgen receptor (AR) inhibitor. Ectopic expression of Wnt-4, $-5 \mathrm{a},-7 \mathrm{~b}$ or -11 in LnCAP, an AR-positive cell line, improves cell survival. Interestingly, enzalutamide increases Wnt-5a expression in this cell line suggesting 
the existence of a positive feedback loop whose knockdown decreases overall cell proliferation [109]. Undoubtedly, examining whether other Wnt/PCP proteins also contribute to drug resistance will deserve further investigation.

\section{Concluding Remarks}

Despite the progress in understanding how Wnt/PCP deregulation contributes to tumorigenesis, many questions remain to be answered. In particular, the functions of many components that constitute this pathway are still poorly understood both at the molecular and cellular levels. Wnt-5a is by far the most studied Wnt/PCP ligand in cancer. However, other less characterized Wnts such as Wnt-2, -7 or -11 are also playing a crucial role in many steps of tumorigenesis and deserve further attention [72, 80, 87]. Moreover, whereas upregulation of $\mathrm{Wnt} / \mathrm{PCP}$ components is frequently associated to poor prognosis and drug resistance, an inverse correlation has also been reported in the literature leading to conflicting conclusions as to whether Wnt-5a act as a tumor suppressor or pro-metastatic factor, very much like TGF $\beta$ [115]. Further contributing to the confusion, Wnt-5a or receptors such as PTK7 have the dual capacity to activate both non-canonical and canonical Wnt pathways. Finally, the frequent repression of canonical Wnt pathway by Wnt/PCP signaling adds an additional layer of complexity. More work is thus needed to further dissect the molecular basis of Wnt/PCP regulation which likely varies with (co)receptors composition at the cell surface (Figure 3) [29].

Given the large body of evidence demonstrating the importance of Wnt/PCP signaling in cancer, this pathway is now considered as an attractive target for novel therapeutics. Different strategies are currently explored using chemical- or antibody-based compounds able to inhibit or activate the pathway and the list of ongoing clinical trials is growing (Table 2). However, development of these therapeutics will certainly highly benefit from the identification of 
reliable biomarkers (assessment of Wnt/PCP component expression levels and/or activation of

335 their associated pathways) able to monitor activation (or inhibition) of the pathway in cells 336 and tissues. Mutations in PRICKLE1 and VANGL1 have been recently described in leukemia 337 [116] and squamous cell carcinoma [117]. Interestingly, these mutations lie in regions 338 important for protein-protein interactions. Yet the relevance of these findings will have to be 339 addressed at the functional and clinical levels. During cancer progression, Wnt/PCP 340 molecules can activate signaling pathways that are not classically associated to Wnt signaling 341 such as Akt [70, 84] or Fyn/Stat [69]. Hijacking of these signaling molecules by Wnt/PCP 342 pathway is likely to render cancer cells more efficient at invading tissues or resisting to 343 chemotherapies. Inhibition of Akt or Fyn with available drugs could thus represent a potential 344 strategy to combat Wnt/PCP deregulation in certain cancers. In conclusion, we believe that 345 therapeutics against Wnt/PCP pathway represent an interesting avenue in cancer treatment. 
$348 \quad$ Figures

$349 \quad$ Figure 1 Wnt signaling pathway and cancer progression

$350 \quad$ Figure 2 Protein composition at the cell surface orients Wnt signaling

352 Table

353 Table 1 Deregulation of non-canonical Wnt/PCP signaling components in cancer

354 Table2: Clinical trials of compounds targeting Wnt/PCP proteins

\section{Text boxes}

357

BOX 1: In 1982, Roel Nusse and Harold Varnus published a Cell paper using Mouse Mammary Tumour Virus (MMTV) to identify genes involved in tumorigenesis. MMTV is a weak oncogenic milk-transmitted retrovirus causing mammary tumours. The authors found that incorporation of MMTV into the mouse genome led to overexpression of a gene christened Int-1 (Integration site-1) correlated to high occurrence of cancer. A few years later, Int-1 was recognized as a homologue of the Drosophila Wingless ligand and renamed Wnt-1. WNT1 was later on defined as an oncogenic protein in humans and found implicated in the $\mathrm{Wnt} / \beta$-catenin pathway.

BOX 2: In the absence of Wnts, $\beta$-catenin is associated to a destruction complex comprising adenomatous polyposis coli (APC), Glycogen Synthase Kinase $3 \beta$ (GSK3 $\beta$ ) and Axin. $\beta$ catenin is phosphorylated by GSK3 $\beta$ leading to its ubiquitylation by SCF $\beta-\operatorname{TrCP}$ and to its degradation by the $26 \mathrm{~S}$ proteasome. In the absence of $\beta$-catenin, the well-defined transcription factors LEF/TCF bind to a repressor called Groucho which recruits histone deacetylases (HDACs) to repress expression of Wnt-target genes. Wnts such as WNT-3A bind to their 
cognate (co)receptors and form a Frizzled-LRP5/6-Wnt ternary complex. Dishevelled is then associated to Frizzled at the plasma membrane resulting in the recruitment of GSK3 $\beta$ and CK1 $\gamma$. Both kinases phosphorylate LRP5/6 at the C-terminus resulting in the formation of high affinity binding sites for Axin. Axin sequestration by LRP5/6 competes with the $\beta$ catenin destruction complex and leads to accumulation of cytosolic $\beta$-catenin and translocation into the nucleus where it displaces Groucho, binds to LEF/TCF and promotes the expression of Wnt-targeted genes.

\section{References:}

1. Nusse, R. and H. Varmus, Three decades of Wnts: a personal perspective on how a scientific field developed. EMBO J, 2012. 31(12): p. 2670-84.

2. Tsukamoto, A.S., et al., Expression of the int-1 gene in transgenic mice is associated with mammary gland hyperplasia and adenocarcinomas in male and female mice. Cell, 1988. 55(4): p. 619-25.

3. Angers, S. and R.T. Moon, Proximal events in Wnt signal transduction. Nat Rev Mol Cell Biol, 2009. 10(7): p. 468-77.

4. Acebron, S.P. and C. Niehrs, beta-Catenin-Independent Roles of Wnt/LRP6 Signaling. Trends Cell Biol, 2016.

5. Anastas, J.N. and R.T. Moon, WNT signalling pathways as therapeutic targets in cancer. Nat Rev Cancer, 2013. 13(1): p. 11-26.

6. Zhan, T., N. Rindtorff, and M. Boutros, Wnt signaling in cancer. Oncogene, 2016.

7. Gubb, D. and A. Garcia-Bellido, A genetic analysis of the determination of cuticular polarity during development in Drosophila melanogaster. J Embryol Exp Morphol, 1982. 68: p. 37-57.

8. Zallen, J.A., Planar polarity and tissue morphogenesis. Cell, 2007. 129(6): p. 105163.

9. Yang, Y. and M. Mlodzik, Wnt-Frizzled/planar cell polarity signaling: cellular orientation by facing the wind (Wnt). Annu Rev Cell Dev Biol, 2015. 31: p. 623-46. 
401

402

403

404

405

406

407

408

409

410

411

412

413

414

415

416

417

418

419

420

421

422

423

424

425

426

427

428

429

430

431

432

433

10. Gray, R.S., I. Roszko, and L. Solnica-Krezel, Planar cell polarity: coordinating morphogenetic cell behaviors with embryonic polarity. Dev Cell, 2011. 21(1): p. 12033.

11. Narimatsu, M., et al., Regulation of planar cell polarity by Smurf ubiquitin ligases. Cell, 2009. 137(2): p. 295-307.

12. Ezan, J. and M. Montcouquiol, Revisiting planar cell polarity in the inner ear. Semin Cell Dev Biol, 2013. 24(5): p. 499-506.

13. McNeill, H., Planar cell polarity: keeping hairs straight is not so simple. Cold Spring Harb Perspect Biol, 2010. 2(2): p. a003376.

14. Sebbagh, M. and J.P. Borg, Insight into planar cell polarity. Exp Cell Res, 2014. 328(2): p. 284-95.

15. Gao, B., Wnt regulation of planar cell polarity (PCP). Curr Top Dev Biol, 2012. 101: p. 263-95.

16. Kibar, Z., et al., Ltap, a mammalian homolog of Drosophila Strabismus/Van Gogh, is altered in the mouse neural tube mutant Loop-tail. Nat Genet, 2001. 28(3): p. 251-5.

17. Kikuchi, A., H. Yamamoto, and S. Kishida, Multiplicity of the interactions of Wnt proteins and their receptors. Cell Signal, 2007. 19(4): p. 659-71.

18. Oishi, I., et al., The receptor tyrosine kinase Ror2 is involved in non-canonical Wnt5a/JNK signalling pathway. Genes Cells, 2003. 8(7): p. 645-54.

19. Niehrs, C., The complex world of WNT receptor signalling. Nat Rev Mol Cell Biol, 2012. 13(12): p. 767-79.

20. Martinez, S., et al., The PTK7 and ROR2 Protein Receptors Interact in the Vertebrate WNT/Planar Cell Polarity (PCP) Pathway. J Biol Chem, 2015. 290(51): p. 30562-72.

21. Podleschny, M., et al., A PTK7/Ror2 Co-Receptor Complex Affects Xenopus Neural Crest Migration. PLoS One, 2015. 10(12): p. e0145169.

22. Green, J., R. Nusse, and R. van Amerongen, The role of Ryk and Ror receptor tyrosine kinases in Wnt signal transduction. Cold Spring Harb Perspect Biol, 2014. 6(2).

23. Kaykas, A., et al., Mutant Frizzled 4 associated with vitreoretinopathy traps wild-type Frizzled in the endoplasmic reticulum by oligomerization. Nat Cell Biol, 2004. 6(1): p. $52-8$.

24. Maurice, P., et al., Molecular organization and dynamics of the melatonin MT(1) receptor/RGS20/G(i) protein complex reveal asymmetry of receptor dimers for $R G S$ and $G(i)$ coupling. EMBO J, 2010. 29(21): p. 3646-59. 
25. Angers, S., et al., Detection of beta 2-adrenergic receptor dimerization in living cells using bioluminescence resonance energy transfer (BRET). Proc Natl Acad Sci U S A, 2000. 97(7): p. 3684-9.

26. Sheldahl, L.C., et al., Dishevelled activates Ca2+flux, PKC, and CamKII in vertebrate embryos. J Cell Biol, 2003. 161(4): p. 769-77.

27. Veeman, M.T., et al., Zebrafish prickle, a modulator of noncanonical Wnt/Fz signaling, regulates gastrulation movements. Curr Biol, 2003. 13(8): p. 680-5.

28. Mikels, A.J. and R. Nusse, Wnts as ligands: processing, secretion and reception. Oncogene, 2006. 25(57): p. 7461-8.

29. Mikels, A.J. and R. Nusse, Purified Wnt5a protein activates or inhibits beta-cateninTCF signaling depending on receptor context. PLoS Biol, 2006. 4(4): p. e115.

30. Puppo, F., et al., Protein tyrosine kinase 7 has a conserved role in Wnt/beta-catenin canonical signalling. EMBO Rep, 2011. 12(1): p. 43-9.

31. Hayes, M., et al., Ptk7 promotes non-canonical Wnt/PCP-mediated morphogenesis and inhibits Wnt/beta-catenin-dependent cell fate decisions during vertebrate development. Development, 2013. 140(8): p. 1807-18.

32. Bin-Nun, N., et al., PTK7 modulates Wnt signaling activity via LRP6. Development, 2014. 141(2): p. 410-21.

33. Chan, D.W., et al., Prickle-1 negatively regulates Wnt/beta-catenin pathway by promoting Dishevelled ubiquitination/degradation in liver cancer. Gastroenterology, 2006. 131(4): p. 1218-27.

34. Cheng, R., et al., Wnt5a suppresses colon cancer by inhibiting cell proliferation and epithelial-mesenchymal transition. J Cell Physiol, 2014. 229(12): p. 1908-17.

35. Liang, H., et al., Wnt5a inhibits $B$ cell proliferation and functions as a tumor suppressor in hematopoietic tissue. Cancer Cell, 2003. 4(5): p. 349-60.

36. Kremenevskaja, N., et al., Wnt-5a has tumor suppressor activity in thyroid carcinoma. Oncogene, 2005. 24(13): p. 2144-54.

37. Dejmek, J., et al., Expression and signaling activity of Wnt-5a/discoidin domain receptor-1 and Syk plays distinct but decisive roles in breast cancer patient survival. Clin Cancer Res, 2005. 11(2 Pt 1): p. 520-8.

38. Bakker, E.R., et al., Wnt5a promotes human colon cancer cell migration and invasion but does not augment intestinal tumorigenesis in Apc1638N mice. Carcinogenesis, 2013. 34(11): p. 2629-38. 
39. Kurayoshi, M., et al., Expression of Wnt-5a is correlated with aggressiveness of gastric cancer by stimulating cell migration and invasion. Cancer Res, 2006. 66(21): p. 10439-48.

40. Lhoumeau, A.C., et al., Overexpression of the Promigratory and Prometastatic PTK7 Receptor Is Associated with an Adverse Clinical Outcome in Colorectal Cancer. PLoS One, 2015. 10(5): p. e0123768.

41. Belotti, E., et al., The human PDZome: a gateway to PSD95-Disc large-zonula occludens (PDZ)-mediated functions. Mol Cell Proteomics, 2013. 12(9): p. 2587-603.

42. Anastas, J.N., et al., A protein complex of SCRIB, NOSIAP and VANGL1 regulates cell polarity and migration, and is associated with breast cancer progression. Oncogene, 2012. 31(32): p. 3696-708.

43. Puvirajesinghe, T.M., et al., Identification of p62/SQSTM1 as a component of noncanonical Wht VANGL2-JNK signalling in breast cancer. Nat Commun, 2016. 7: p. 10318.

44. Asad, M., et al., FZD7 drives in vitro aggressiveness in Stem-A subtype of ovarian cancer via regulation of non-canonical Wnt/PCP pathway. Cell Death Dis, 2014. 5: p. e1346.

45. Bilder, D., M. Li, and N. Perrimon, Cooperative regulation of cell polarity and growth by Drosophila tumor suppressors. Science, 2000. 289(5476): p. 113-6.

46. Montcouquiol, M., et al., Identification of Vangl2 and Scrb1 as planar polarity genes in mammals. Nature, 2003. 423(6936): p. 173-7.

47. Feigin, M.E., et al., Mislocalization of the cell polarity protein scribble promotes mammary tumorigenesis and is associated with basal breast cancer. Cancer Res, 2014. 74(11): p. 3180-94.

48. Pearson, H.B., et al., SCRIB expression is deregulated in human prostate cancer, and its deficiency in mice promotes prostate neoplasia. J Clin Invest, 2011. 121(11): p. 4257-67.

49. Zhan, L., et al., Deregulation of scribble promotes mammary tumorigenesis and reveals a role for cell polarity in carcinoma. Cell, 2008. 135(5): p. 865-78.

50. Cordenonsi, M., et al., The Hippo transducer TAZ confers cancer stem cell-related traits on breast cancer cells. Cell, 2011. 147(4): p. 759-72.

51. Godde, N.J., et al., Scribble modulates the MAPK/Fral pathway to disrupt luminal and ductal integrity and suppress tumour formation in the mammary gland. PLoS Genet, 2014. 10(5): p. e1004323. 
52. Caddy, J., et al., Epidermal wound repair is regulated by the planar cell polarity signaling pathway. Dev Cell, 2010. 19(1): p. 138-47.

53. Darido, C., et al., Targeting of the tumor suppressor GRHL3 by a miR-21-dependent proto-oncogenic network results in PTEN loss and tumorigenesis. Cancer Cell, 2011. 20(5): p. 635-48.

54. Lee, H. and P.N. Adler, The grainy head transcription factor is essential for the function of the frizzled pathway in the Drosophila wing. Mech Dev, 2004. 121(1): p. 37-49.

55. Mace, K.A., J.C. Pearson, and W. McGinnis, An epidermal barrier wound repair pathway in Drosophila is mediated by grainy head. Science, 2005. 308(5720): p. 3815.

56. Landeira, D., et al., Jarid2 Coordinates Nanog Expression and PCP/Wnt Signaling Required for Efficient ESC Differentiation and Early Embryo Development. Cell Rep, 2015. 12(4): p. 573-86.

57. Tange, S., et al., JARID2 is involved in transforming growth factor-beta-induced epithelial-mesenchymal transition of lung and colon cancer cell lines. PLoS One, 2014. 9(12): p. e115684.

58. Lei, X., et al., JARID2 promotes invasion and metastasis of hepatocellular carcinoma by facilitating epithelial-mesenchymal transition through PTEN/AKT signaling. Oncotarget, 2016.

59. Lhoumeau, A.C., et al., Ptk7-Deficient Mice Have Decreased Hematopoietic Stem Cell Pools as a Result of Deregulated Proliferation and Migration. J Immunol, 2016. 196(10): p. 4367-77.

60. Jung, P., et al., Isolation of Human Colon Stem Cells Using Surface Expression of PTK7. Stem Cell Reports, 2015. 5(6): p. 979-87.

61. Gonzalez, D.M. and D. Medici, Signaling mechanisms of the epithelial-mesenchymal transition. Sci Signal, 2014. 7(344): p. re8.

62. Taki, M., et al., Down-regulation of Wnt-4 and up-regulation of Wnt-5a expression by epithelial-mesenchymal transition in human squamous carcinoma cells. Cancer Sci, 2003. 94(7): p. 593-7.

63. Chen, Y.S., et al., Proteomics profiling of Madin-Darby canine kidney plasma membranes reveals Wnt-5a involvement during oncogenic H-Ras/TGF-beta-mediated epithelial-mesenchymal transition. Mol Cell Proteomics, 2011. 10(2): p. M110 001131. 
64. Gupta, S., et al., FZD4 as a mediator of ERG oncogene-induced WNT signaling and epithelial-to-mesenchymal transition in human prostate cancer cells. Cancer Res, 2010. 70(17): p. 6735-45.

65. Chan, D.N., et al., PTK7 marks the first human developmental EMT in vitro. PLoS One, 2012. 7(11): p. e50432.

66. Dissanayake, S.K., et al., The Wnt5A/protein kinase C pathway mediates motility in melanoma cells via the inhibition of metastasis suppressors and initiation of an epithelial to mesenchymal transition. J Biol Chem, 2007. 282(23): p. 17259-71.

67. Yamben, I.F., et al., Scrib is required for epithelial cell identity and prevents epithelial to mesenchymal transition in the mouse. Dev Biol, 2013. 384(1): p. 41-52.

68. Elsum, I.A., C. Martin, and P.O. Humbert, Scribble regulates an EMT polarity pathway through modulation of MAPK-ERK signaling to mediate junction formation. J Cell Sci, 2013. 126(Pt 17): p. 3990-9.

69. Gujral, T.S., et al., A noncanonical Frizzled2 pathway regulates epithelialmesenchymal transition and metastasis. Cell, 2014. 159(4): p. 844-56.

70. Daulat, A.M., et al., PRICKLE1 Contributes to Cancer Cell Dissemination through Its Interaction with mTORC2. Dev Cell, 2016. 37(4): p. 311-25.

71. Lim, B.C., et al., Prickle1 promotes focal adhesion disassembly in cooperation with the CLASP-LL5beta complex in migrating cells. J Cell Sci, 2016. 129(16): p. 3115-29.

72. Luga, V., et al., Exosomes mediate stromal mobilization of autocrine Wnt-PCP signaling in breast cancer cell migration. Cell, 2012. 151(7): p. 1542-56.

73. Weeraratna, A.T., et al., Wnt5a signaling directly affects cell motility and invasion of metastatic melanoma. Cancer Cell, 2002. 1(3): p. 279-88.

74. Da Forno, P.D., et al., WNT5A expression increases during melanoma progression and correlates with outcome. Clin Cancer Res, 2008. 14(18): p. 5825-32.

75. Dissanayake, S.K., et al., Wnt5A regulates expression of tumor-associated antigens in melanoma via changes in signal transducers and activators of transcription 3 phosphorylation. Cancer Res, 2008. 68(24): p. 10205-14.

76. O'Connell, M.P., et al., The orphan tyrosine kinase receptor, ROR2, mediates Wnt5A signaling in metastatic melanoma. Oncogene, 2010. 29(1): p. 34-44.

77. Witze, E.S., et al., Wnt5a control of cell polarity and directional movement by polarized redistribution of adhesion receptors. Science, 2008. 320(5874): p. 365-9.

78. Witze, E.S., et al., Wnt5a directs polarized calcium gradients by recruiting cortical endoplasmic reticulum to the cell trailing edge. Dev Cell, 2013. 26(6): p. 645-57. 
79. Matsumoto, S., et al., Binding of APC and dishevelled mediates Wnt5a-regulated focal adhesion dynamics in migrating cells. EMBO J, 2010. 29(7): p. 1192-204.

80. Yu, M., et al., RNA sequencing of pancreatic circulating tumour cells implicates WNT signalling in metastasis. Nature, 2012. 487(7408): p. 510-3.

81. Hatakeyama, J., et al., Vangll and Vangl2: planar cell polarity components with a developing role in cancer. Endocr Relat Cancer, 2014. 21(5): p. R345-56.

82. Jenny, A., et al., Prickle and Strabismus form a functional complex to generate a correct axis during planar cell polarity signaling. EMBO J, 2003. 22(17): p. 4409-20.

83. Zhang, L., et al., A lateral signalling pathway coordinates shape volatility during cell migration. Nat Commun, 2016. 7: p. 11714.

84. Anastas, J.N., et al., WNT5A enhances resistance of melanoma cells to targeted BRAF inhibitors. J Clin Invest, 2014. 124(7): p. 2877-90.

85. Lejeune, S., et al., Wnt5a cloning, expression, and up-regulation in human primary breast cancers. Clin Cancer Res, 1995. 1(2): p. 215-22.

86. MacMillan, C.D., et al., Stage of breast cancer progression influences cellular response to activation of the WNT/planar cell polarity pathway. Sci Rep, 2014. 4: p. 6315.

87. Avgustinova, A., et al., Tumour cell-derived Wnt7a recruits and activates fibroblasts to promote tumour aggressiveness. Nat Commun, 2016. 7: p. 10305.

88. Navarro, C., et al., Junctional recruitment of mammalian Scribble relies on Ecadherin engagement. Oncogene, 2005. 24(27): p. 4330-9.

89. Gao, B., et al., Wht signaling gradients establish planar cell polarity by inducing Vangl2 phosphorylation through Ror2. Dev Cell, 2011. 20(2): p. 163-76.

90. Kelly, L.K., et al., Frizzled-Induced Van Gogh Phosphorylation by CKlepsilon Promotes Asymmetric Localization of Core PCP Factors in Drosophila. Cell Rep, 2016. 16(2): p. 344-56.

91. Dyberg, C., et al., Planar cell polarity gene expression correlates with tumor cell viability and prognostic outcome in neuroblastoma. BMC Cancer, 2016. 16: p. 259.

92. Cantrell, V.A. and J.R. Jessen, The planar cell polarity protein Van Gogh-Like 2 regulates tumor cell migration and matrix metalloproteinase-dependent invasion. Cancer Lett, 2010. 287(1): p. 54-61.

93. Mossie, K., et al., Colon carcinoma kinase-4 defines a new subclass of the receptor tyrosine kinase family. Oncogene, 1995. 11(10): p. 2179-84. 
602

603

604

605

606

607

608

609

610

611

612

613

614

615

616

617

618

619

620

621

622

623

624

625

626

627

628

629

630

631

632

633

634

94. Jung, J.W., et al., Cloning and characterization of the full-length mouse Ptk7 cDNA encoding a defective receptor protein tyrosine kinase. Gene, 2004. 328: p. 75-84.

95. Lhoumeau, A.C., et al., PTK7: a cell polarity receptor with multiple facets. Cell Cycle, 2011. 10(8): p. 1233-6.

96. Prebet, T., et al., The cell polarity PTK7 receptor acts as a modulator of the chemotherapeutic response in acute myeloid leukemia and impairs clinical outcome. Blood, 2010. 116(13): p. 2315-23.

97. Jiang, G., et al., PTK7: a new biomarker for immunophenotypic characterization of maturing T cells and T cell acute lymphoblastic leukemia. Leuk Res, 2012. 36(11): p. 1347-53.

98. Kaucka, M., et al., The planar cell polarity pathway drives pathogenesis of chronic lymphocytic leukemia by the regulation of B-lymphocyte migration. Cancer Res, 2013. 73(5): p. 1491-501.

99. Janovska, P., et al., Autocrine Signaling by Wnt-5a Deregulates Chemotaxis of Leukemic Cells and Predicts Clinical Outcome in Chronic Lymphocytic Leukemia. Clin Cancer Res, 2016. 22(2): p. 459-69.

100. Yu, J., et al., Wht5a induces ROR1/ROR2 heterooligomerization to enhance leukemia chemotaxis and proliferation. J Clin Invest, 2016. 126(2): p. 585-98.

101. Daneshmanesh, A.H., et al., Monoclonal antibodies against RORI induce apoptosis of chronic lymphocytic leukemia (CLL) cells. Leukemia, 2012. 26(6): p. 1348-55.

102. Linke, F., et al., Microenvironmental interactions between endothelial and lymphoma cells: a role for the canonical WNT pathway in Hodgkin lymphoma. Leukemia, 2016.

103. Sun, Y., et al., Treatment-induced damage to the tumor microenvironment promotes prostate cancer therapy resistance through WNT16B. Nat Med, 2012. 18(9): p. 135968.

104. Stanganello, E. and S. Scholpp, Role of cytonemes in Wht transport. J Cell Sci, 2016. 129(4): p. 665-72.

105. Desrochers, L.M., M.A. Antonyak, and R.A. Cerione, Extracellular Vesicles: Satellites of Information Transfer in Cancer and Stem Cell Biology. Dev Cell, 2016. 37(4): p. 301-9.

106. Ekstrom, E.J., et al., WNT5A induces release of exosomes containing pro-angiogenic and immunosuppressive factors from malignant melanoma cells. Mol Cancer, 2014. 13: p. 88 . 
635

636

637

638

639

640

641

642

643

644

645

646

647

648

649

650

651

652

653

654

655

656

657

658

659

660

661

662

663

107. O'Connell, M.P., et al., Hypoxia induces phenotypic plasticity and therapy resistance in melanoma via the tyrosine kinase receptors ROR1 and ROR2. Cancer Discov, 2013. 3(12): p. 1378-93.

108. Ataseven, B., et al., PTK7 expression in triple-negative breast cancer. Anticancer Res, 2013. 33(9): p. 3759-63.

109. Miyamoto, D.T., et al., RNA-Seq of single prostate CTCs implicates noncanonical Wnt signaling in antiandrogen resistance. Science, 2015. 349(6254): p. 1351-6.

110. Safholm, A., et al., The Wnt-5a-derived hexapeptide Foxy-5 inhibits breast cancer metastasis in vivo by targeting cell motility. Clin Cancer Res, 2008. 14(20): p. 655663.

111. Jonsson, M., et al., Loss of Wnt-5a protein is associated with early relapse in invasive ductal breast carcinomas. Cancer Res, 2002. 62(2): p. 409-16.

112. Phesse, T., D. Flanagan, and E. Vincan, Frizzled7: A Promising Achilles' Heel for Targeting the Wnt Receptor Complex to Treat Cancer. Cancers (Basel), 2016. 8(5).

113. Steinhart, Z., et al., A CRISPR screen reveals a WNT7B-FZD5 signaling circuit as a therapeutic opportunity in pancreatic cancer. bioRxiv, 2016.

114. Masuda, M., et al., TNIK inhibition abrogates colorectal cancer stemness. Nat Commun, 2016. 7: p. 12586.

115. Katsuno, Y., S. Lamouille, and R. Derynck, TGF-beta signaling and epithelialmesenchymal transition in cancer progression. Curr Opin Oncol, 2013. 25(1): p. 7684.

116. Wang, L., et al., Somatic mutation as a mechanism of Wnt/beta-catenin pathway activation in CLL. Blood, 2014. 124(7): p. 1089-98.

117. Qin, H.D., et al., Genomic Characterization of Esophageal Squamous Cell Carcinoma Reveals Critical Genes Underlying Tumorigenesis and Poor Prognosis. Am J Hum Genet, 2016. 98(4): p. 709-27. 
665 A. Wnts bind either to Frizzled receptors or to co-receptors leading to activation of diverse 666 intracellular signaling pathways including $\mathrm{Wnt} / \mathrm{Ca}^{2+}$ or $\mathrm{Wnt} / \mathrm{\beta}$-catenin and $\mathrm{Wnt} / \mathrm{PCP}$ which

667

668 acts through the activation of small G-proteins such as Rho, Rac and Cdc42. Signaling molecules with unknown function in Wnt signaling are in light green.

\section{B. Top panel. Development and progression of epithelium-derived cancer.}

After transformation of the cell of origin (green) (a.), cancer stem cells (red) start to proliferate (b.). Cells escape from the site of the primary tumour after gaining cell motility and invasive abilities (c.). Circulating stem cells (red) found in the bloodstream and the lymphatic system invade distal organs and prime sites of metastasis by activating fibroblasts (e.). Some cells can resist to chemotherapy and contribute to patient relapse (f.).

Bottom panel. Contribution of Wnt/ $\beta$-catenin and Wnt/PCP signaling in the progression of cancer disease. Wnt/ $\beta$-catenin signaling occurs at the early stage of cancer progression providing proliferative cues to cells. Wnt/ $\beta$-catenin signaling can be inhibited by components of Wnt/PCP signaling. During cancer progression, a shift of Wnt signaling occurs: Wnt/PCP signaling takes over and contributes to cancer progression by increasing cell motility, invasion, priming metastasis niche and resistance to therapy.

\section{FIGURE 2: Protein composition at cell surface orients Wnt signaling}

Depending on a particular cellular context (A or B), a given Wnt binds to Frizzled and its coreceptors and activates downstream signaling pathways leading to A or B signaling.

\section{Acknowledgments}

We would like to sincerely apologize to our colleagues whose contributions were not cited in this review due to manuscript length limitation. The authors wish to thank Eric Bailly, 
689 Stéphane Angers and Valerie Ferrier for critical review of the manuscript. JP Borg's lab is 690 funded by La Ligue Nationale Contre le Cancer (Label Ligue J.P.B.), Fondation de France 691 (post-doctoral fellowship to A.M.D.), Fondation ARC pour la Recherche sur le Cancer, Ruban 692 Rose and SIRIC (INCa-DGOS-Inserm 6038, fellowship to A.M.D.). JPB is a scholar of 693 Institut Universitaire de France.

694 
Table 1: Deregulation of non-canonical Wnt/PCP signaling components in cancer

\begin{tabular}{llll}
\hline Type of cancer & Name of the genes & Features & References \\
\hline \hline Breast & WNT-5A & Increased tumorigenesis & Lejeune S et al., 1995; MacMillan, C.D., et \\
& & al.,2014 \\
& WNT-11 & Increased tumorigenesis & Avgustinova et al., 2016 \\
WNT-7A & Increased tumorigenesis & Luga V et al., 2012 \\
FRIZZLED-7 & Increased tumorigenesis & Yang et al., 2011 \\
& VANGL-1 & Increased tumorigenesis & Anastas et al., 2012 \\
& VANGL-2 & Increased tumorigenesis & Purivajessinghe et al., 2016 \\
SCRIBBLE & Increased tumorigenesis & Anastas et al., 2012 \\
PRICKLE-1 & Increased tumorigenesis & Daulat et al., 2016 \\
FRIZZLED-2 & Increased tumorigenesis & Gujral et al., 2014 \\
PTK-7 & Increased tumorigenesis & Gärtner et al., 2014 \\
ROR2 & Increased tumorigenesis & Henry et al., 2015 \\
PTK7 & Increased resistance to therapy & Ataseven et al., 2013 \\
& &
\end{tabular}

\begin{tabular}{llll}
\hline \hline Ovarian & FRIZZLED-7 & Increased tumorigenesis & Asad et al., 2014 \\
& & & \\
\hline \hline Squamous carcinoma & WNT-5A & Increased tumorigenesis & Taki et al., 2003 \\
& VANGL-1 & Increased tumorigenesis & Qin et al., 2016
\end{tabular}

\begin{tabular}{llll}
\hline \hline Neuroblastoma & VANGL-2 & Decreased tumorigenesis & Dyberg et al., 2016 \\
& PRICKLE-1 & Decreased tumorigenesis & Dyberg et al., 2016
\end{tabular}

\begin{tabular}{llll}
\hline \hline Melanoma & & \\
& WNT-5A & Increased tumorigenesis & Dissanyake et al., 2007 \\
WNT-5A & Increased tumorigenesis & Weeraratna et al., 2002 \\
WNT-5A & Increased tumorigenesis & Da Forno et al., 2008 \\
WNT-5A & Increased resistance to therapy & Anastas et al., 2014 \\
FRIZZLED-7 & Increased tumorigenesis & Tiwary et al., Plos One 2016 \\
FRIZZLED-7 & Increased resistance to therapy & Anastas et al., JCI 2014 \\
& SFRP3 & Methylation, loss of expression. & Ekström et al., Plos One 2011
\end{tabular}


Decreased tumorigenesis

$\begin{array}{lll}\text { FRIZZLED-5 } & \text { Increased tumorigenesis } & \text { Weeraratna et al., } 2002 \\ \text { ROR2 } & \text { Increased tumorigenesis } & \text { O'Connell et al., Oncogene } 2010 \\ \text { ROR1 } & \text { Increased tumorigenesis } & \text { O'Connell et al., Cancer Discovery } 2013\end{array}$

\begin{tabular}{llll}
\hline \hline Colorectal & PTK7 & Increased tumorigenesis & Lhoumeau et al., Plos One 2015 \\
ROR2 & Increased tumorigenesis & Mei et al., BBRC 2014 \\
WNT-5A & Tumor suppressor & Cheng et al., J Cell Physiology 2014 \\
WNT-5A & Increased tumorigenesis & Bakker et al., 2013
\end{tabular}

\begin{tabular}{llll}
\hline \hline Gastric cancer & WNT-5A & Increased tumorigenesis & Kurayoshi et al., 2006 \\
& FRIZZLED-5 & Increased tumorigenesis & Steinhart et al., Biorxiv.org \\
& FRIZZLED-4 & Increased tumorigenesis & Gupta et al., 2010 \\
Pancreas & Increased tumorigenesis & Yu et al. 2012 \\
& WNT-5A & Increased resistance to therapy & Miyato et al., 2015 \\
& & & Kremenevskaja et al., 2005
\end{tabular}

Leukemia

CLL

$\begin{array}{lll}\text { PRICKLE1 } & \text { Increased tumorigenesis } & \text { Kaucka et al., } 2013 \\ \text { VANGL2 } & \text { Increased tumorigenesis } & \text { Kaucka et al., } 2013 \\ \text { CELSR1 } & \text { Increased tumorigenesis } & \text { Kaucka et al., } 2013 \\ \text { Frizzled-3 } & \text { Increased tumorigenesis } & \text { Kaucka et al., } 2013 \\ \text { Frizzled-7 } & \text { Increased tumorigenesis } & \text { Kaucka et al., } 2013 \\ \text { Dishevelled 2 } & \text { Increased tumorigenesis } & \text { Kaucka et al., } 2013 \\ \text { Dishevelled 3 } & \text { Increased tumorigenesis } & \text { Kaucka et al., } 2013 \\ \text { Casein kinase 1 } & \text { Increased tumorigenesis } & \text { Kaucka et al., } 2013 \\ \text { ROR1 } & \text { Increased tumorigenesis } & \text { Janovska et al., } 2016 \\ \text { WNT-5A } & \text { Increased tumorigenesis } & \text { Kaucka et al., } 2013 \\ & \text { Increased tumorigenesis } & \text { Yu et al., 2016 } \\ \text { WNT-5A } & \text { Tumor suppressor } & \text { Liang, H et al., } 2003\end{array}$

$A M L$

PTK7

Increased tumorigenesis

Prebet et al., Blood 2010 
Table 2: Clinical trials of compounds targeting Wnt/PCP proteins

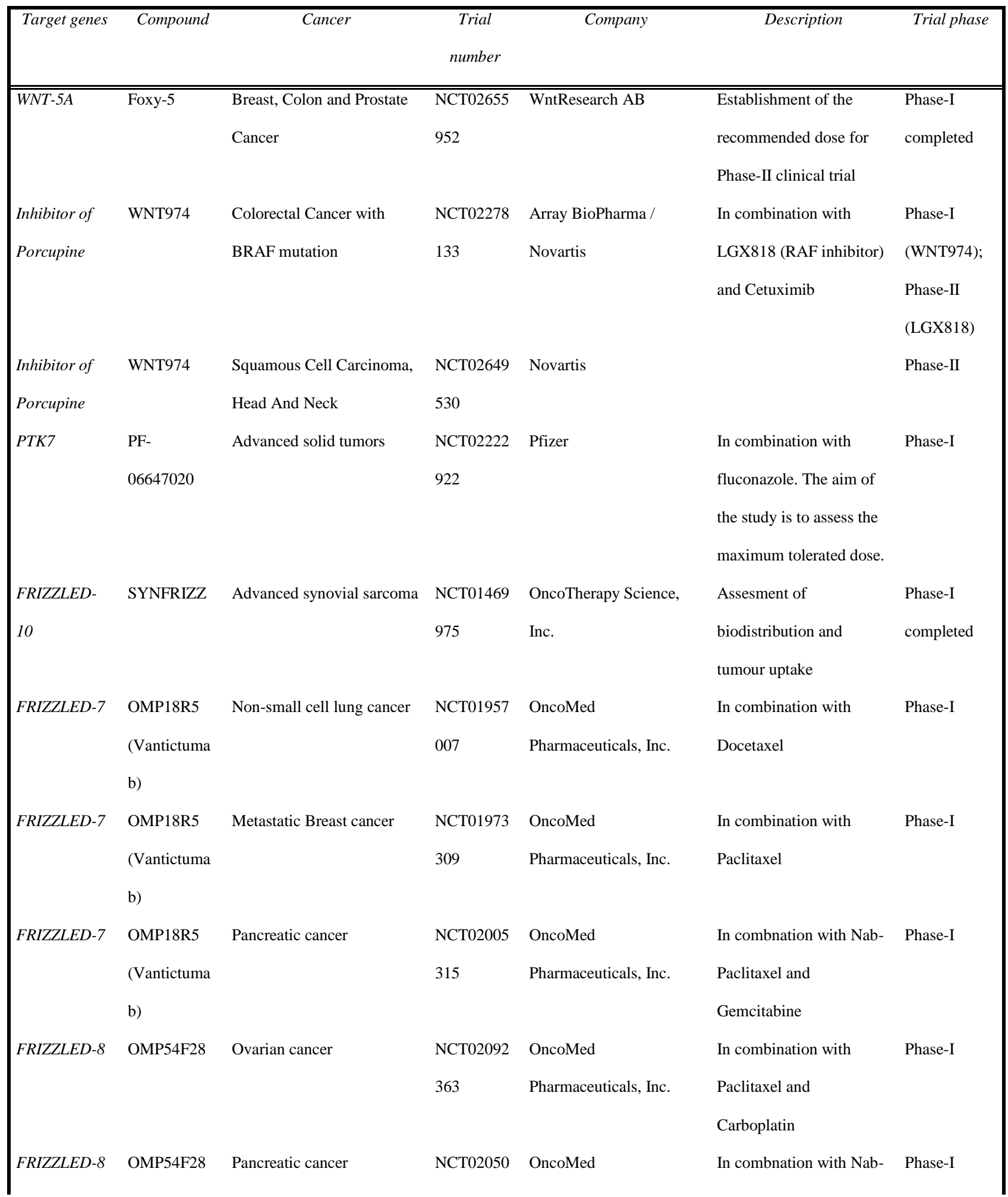




\section{Outstanding questions box}

- How does the Wnt/PCP pathway function in normal and cancer cells?

- What are the mechanisms that localize and regulate Wnt/PCP molecules at the plasma membrane?

- What is the best readout to monitor Wnt/PCP activation in normal and cancer cells?

- What are the mechanisms that up-regulate expression of Wnt/PCP molecules in cancer cells?

- Are Wnt ligands and receptors mandatory to trigger abnormal activation of Wnt/PCP signaling in cancer cells?

- Which Wnt/PCP components should be targeted in priority in tumors and how?

- Is potential upregulation of canonical Wnt pathway activity upon inhibition of the Wnt/PCP pathway a problem?

- Is it possible to anticipate the side effects of therapeutic inhibition of Wnt/PCP pathway? 


\section{FIGURE 1:}

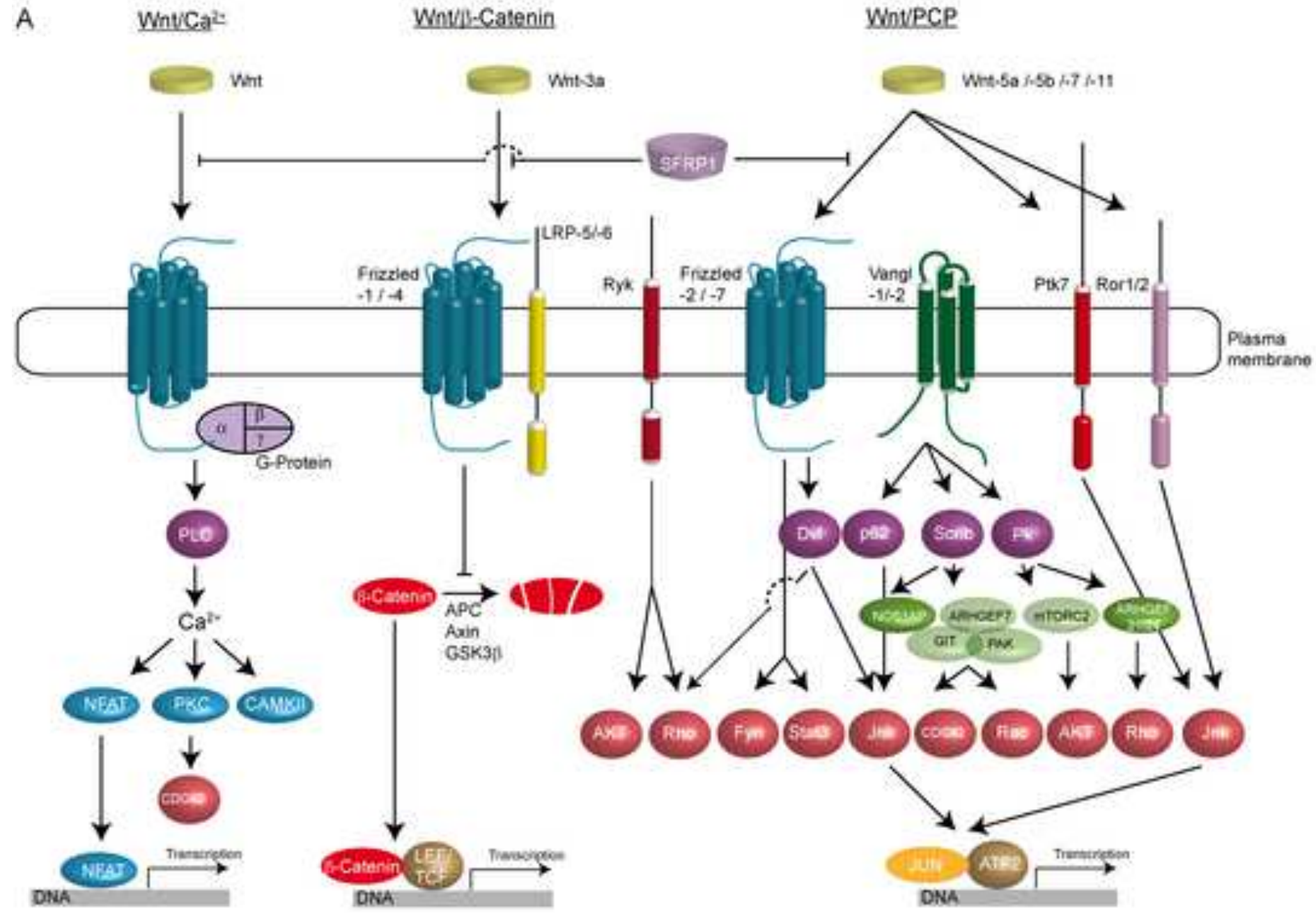

B

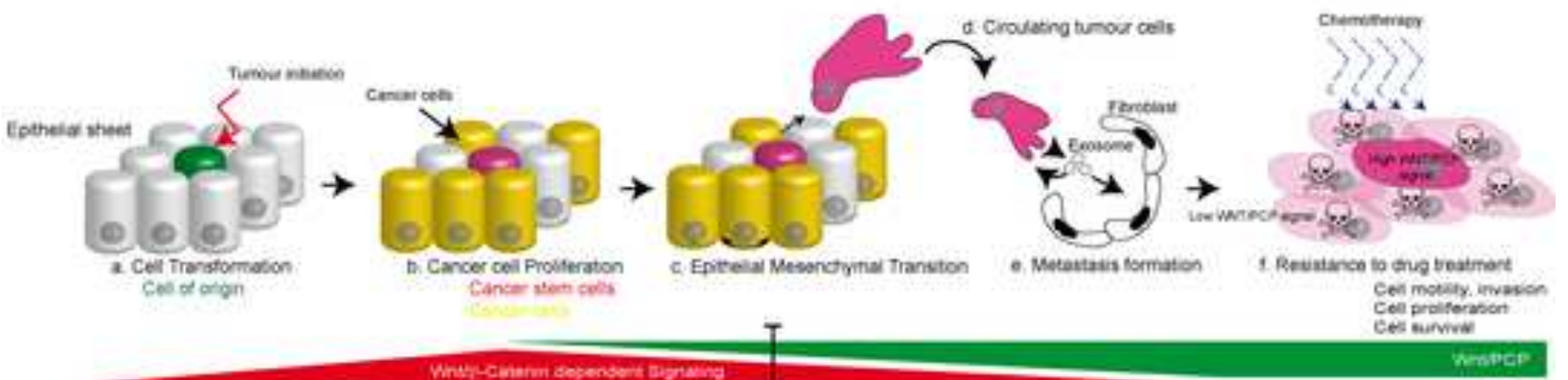




\section{FIGURE 2:}

Cellular context $\mathrm{A}$

Cellular context B

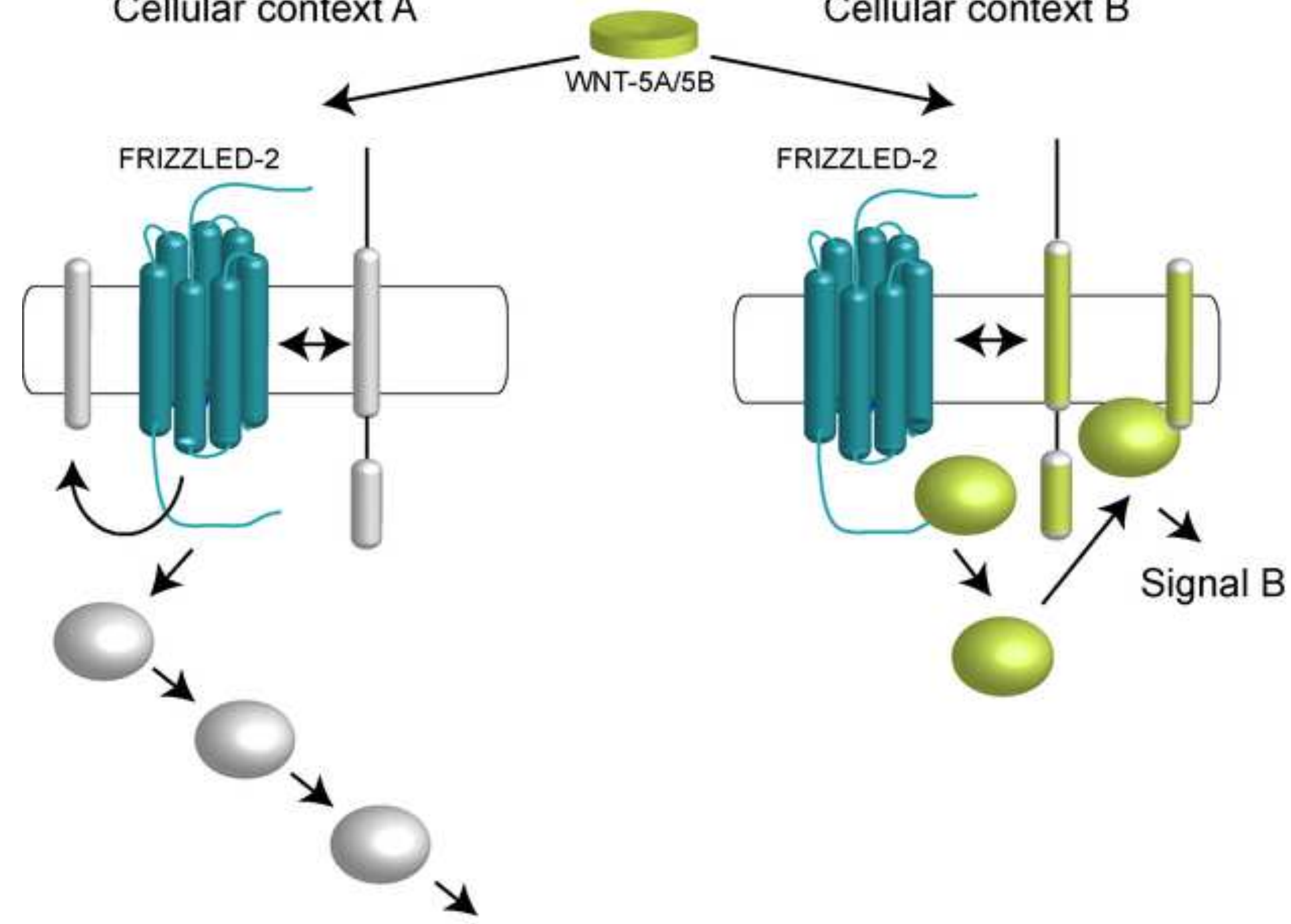

Signal A 